Revista Complutense de Educación

ISSNe: 1988-2793

http://dx.doi.org/10.5209/RCED.57160

\title{
Análisis descriptivos de datos con IBM SPSS Statistics
}

\section{Autores: Ana $\mathrm{M}^{\mathrm{a}}$. Porto Castro y $\mathrm{M}^{\mathrm{a}}$. Josefa Mosteiro García}

Editorial: Andavira

Año de publicación: 2016

$\mathrm{N}^{\mathrm{a}}$ de páginas: 149

ISBN: 978-84-8408-936-0

Análisis descriptivos de datos con IBM SPSS Statistics, es una obra reciente y actual del año 2016, escrita por Ana $\mathrm{M}^{\mathrm{a}}$ Porto Castro y $\mathrm{M}^{\mathrm{a}}$ Josefa Mosteiro García. Donde a través del uso de un lenguaje sencillo y directo presentan una guía básica de estadísticas descriptiva organizada en 6 capítulos teóricos acompañados casi todos ellos, a excepción del capítulo dos referido al funcionamiento general del programa SPSS, de actividades prácticas. Por el contenido de los ejemplos expuesto en la obra y las cuestiones planteadas, es una obra dirigida especialmente a estudiantes del campo de la educación, pero los conceptos explicados son extrapolables a cualquier otra disciplina.

A través de dicho libro se hace un recorrido por toda la estadística descriptiva, desde los conceptos más básicos tales como, población, muestra, variables, etc., a algunos más complejos como pueden ser los tipos de correlación: correlación de Pearson, de Spearman, coeficiente phi, etc. La estructura del libro es básicamente la misma en casi todos los capítulos; se explica de manera teórica los distintos conceptos y después se muestra cómo representarlo mediante el SPSS. Este mix de teoría y práctica, acompañado de explicaciones sencillas, adaptadas a un público que está comenzando en el estudio de esta temática, da lugar a una lectura amena fácil de comprender.

La obra comienza con un capítulo de conceptos básicos a través del cual se pretende introducir al lector en el campo de la estadística elemental. Para ello se explican aspectos tan fundamentales como la clasificación de las variables, qué es la medición, los tipos de escala de medidas, etc., hasta finalizar el capítulo con una serie de actividades prácticas en las que se plantean cuestiones relacionadas con los conceptos estudiados. En el capítulo dos se introduce al lector en el manejo del programa estadísticos SPSS. Las autoras van orientando al mismo en todo momento, desde aspectos tan básicos como los pasos a seguir para entrar al programa, explicación de las distintas ventanas, explicación de la barra del menú, etc., hasta acciones tan útiles como la introducción de datos, fusión de archivos, selección de caso, etc. Este capítulo es quizás el más teórico de todos, pero al mismo tiempo el más explicativo para aquellas personas que no tienen muchos conocimientos sobre dicho programa, ya que se explican las funciones más fundamentales para un manejo correcto del SPSS. En el siguiente capítulo se muestra cómo organizar los datos y cómo representarlos, por ejemplo, primero se explica lo que son las tablas de distribuciones o los distintos tipos de gráficos y después de manera práctica, cómo hacer estas tablas y los gráficos mediante el programa de análisis de datos. Aquí las actividades prácticas 
que se plantean requieren del manejo del SPSS, lo que supone el primer contacto del lector con el mismo. El capítulo cuatro trata sobre las medidas descriptivas básicas, distinguiendo entre las medidas de tendencia central, las medidas de dispersión o de variabilidad y los índices de forma, asimetría y curtosis. En este capítulo debido a la carga matemática que conlleva la compresión del mismo, se ponen ejemplos prácticos resueltos de los conceptos explicados. En cuanto al capítulo cinco, sobre las puntuaciones individuales y la curva normal, los conceptos se vuelven algo más complejos para aquellos lectores con escasos conocimientos estadísticos. Quizás el acercamiento que se realiza a la explicación de la distribución normal y las áreas bajo la curva normal sea lo más difícil de entender en esta parte, pero gracias al uso de figuras, tablas y de un lenguaje específico mediante el cual se explican los aspectos más básicos sobre este tema, sin llegar a profundizar mucho en el mismo, el lector puede comprenderlo sin dificultad. Finalmente la obra se cierra con el capítulo seis donde se habla de las relaciones entre las variables, concretamente sobre los tipos de correlación y la realización e interpretación de las mismas con el SPSS. Hay que señalar que la obra termina con este capítulo y no se añade ningún apartado ni de conclusiones finales ni de referencias bibliográficas; las cuales podrían servir de guía al lector para profundizar en mucho de los conceptos explicados en la obra.

Algo positivo de este libro es que no es muy extenso ni intenso a nivel teórico, y además no realiza un uso excesivo de tecnicismos complejos relacionados con el contenido explicado. Otro aspecto a destacar es que, tal y como se ha comentado, al final de casi todos los capítulos se presentan una serie de actividades que le permite al lector evaluar sus propios conocimientos sobre los contenidos explicados. Estas actividades no son muy numerosas y no requieren de mucho tiempo para su realización ya que son enunciados muy concretos carentes de ambigüedad. El único problema de estas cuestiones planteadas es que no aparecen resueltas a lo largo de la obra, lo que limita la capacidad del lector para conocer si los problemas los ha realizado correctamente y por ende, saber cuál es su nivel de compresión sobre los contenidos estudiados.

A pesar de estos pros y contras, el libro es una guía excelente para aquellos que quieran introducirse en el conocimiento de la estadísticas descriptiva y el manejo del programa SPSS, puesto que para su entendimiento no se requiere del dominio previo de complejos conocimientos estadísticos y/o informáticos, debido al empleo, por parte de las autoras, de un lenguaje explicativo y sencillo que hace que sea comprensible para cualquier lector con interés en la materia.

Delia Arroyo Resino deliaarroyoresino@gmail.com Universidad Complutense de Madrid 